$$
\begin{aligned}
& \text { مجله علوم آمارى، بهار و تابستان IF }
\end{aligned}
$$

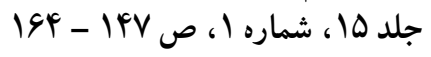

DOI: $10.29252 /$ jss.15.1.23

مقاله بُروهشى

مدل توأم دادههاى شمارشى طولى و زمان تا رخداد بِيشامد صفر آماسيده با استفاده از مدل

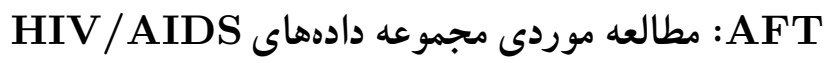

$$
\begin{aligned}
& \text { مجتبى زينلى نجفآبادى، احسان بهرامى سامانى } \\
& \text { كروه آمار، دانشكده علوم رياضى، دانشكاه شهيد بهشتى }
\end{aligned}
$$

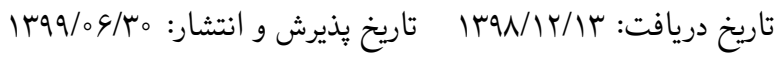

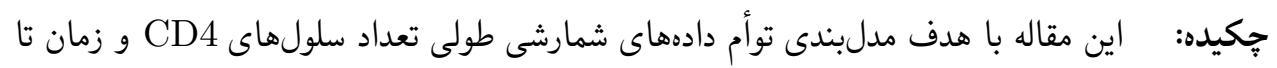

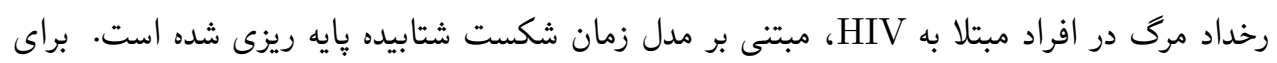

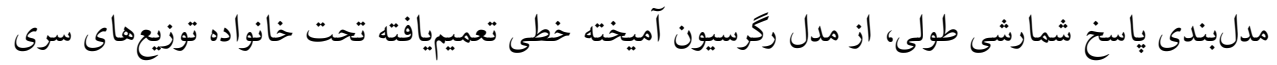

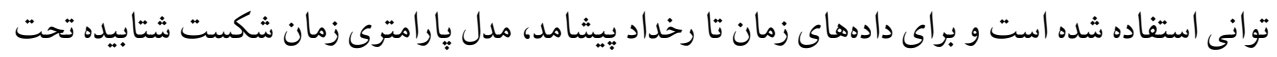

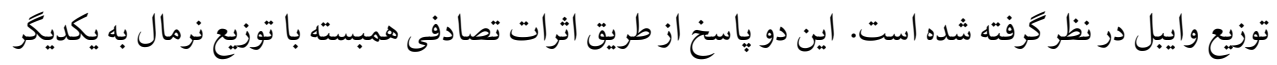

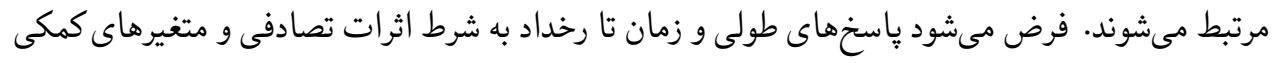

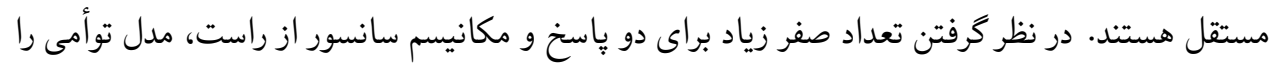

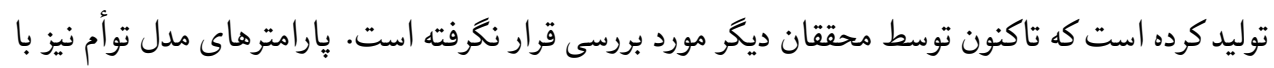
روشهاى مونت كارلوى زنجير ماركوفى برآورد شدند.

وازههاى كليدى: مدل زمان شكست شتابيده، زمان تا رخداد بيشامد، سانسور از راست، خانواده توزيعهاى سرى توانى، مدل ركرسيون آميخته خطى تعميميافته.

آدرس الكترونيكى نويسنده مسئول مقاله: احسان بهرامى سامانى،،

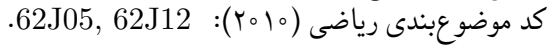


مدل توأم دادههاى شمارشى طولى و زمان

IYA

\section{|}

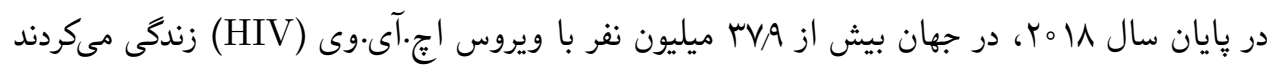

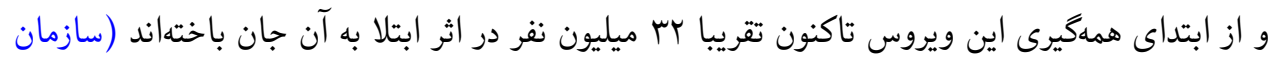

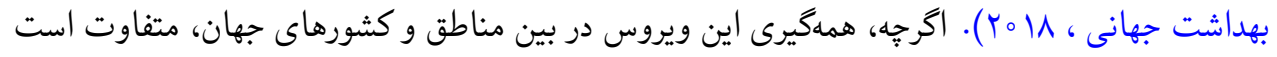

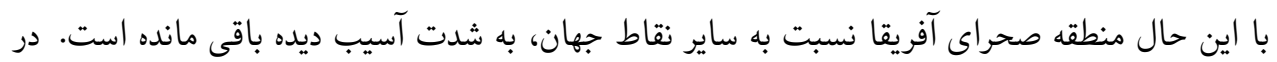

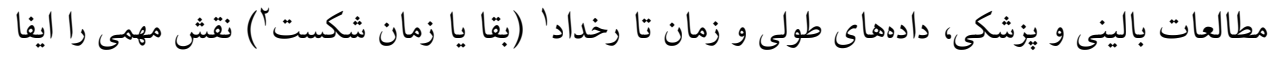

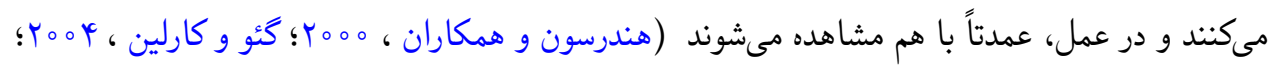

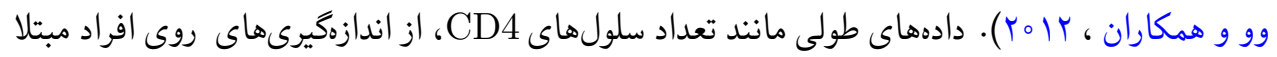

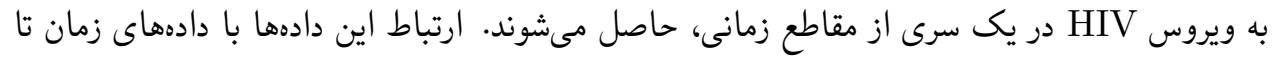

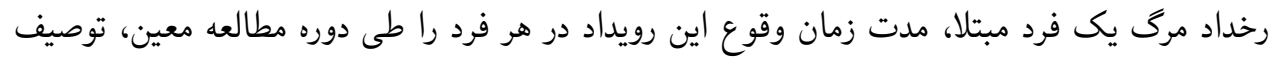

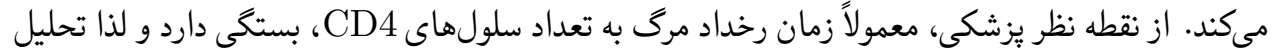

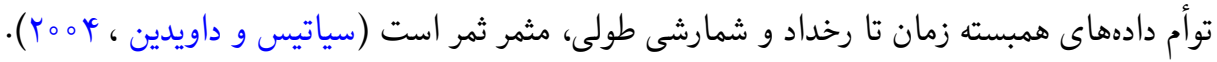

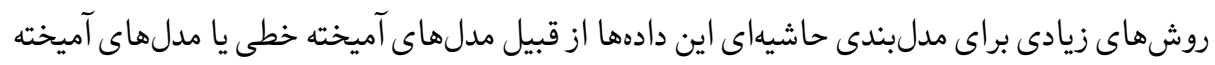

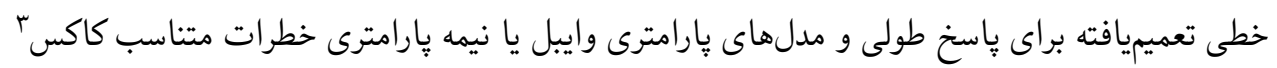

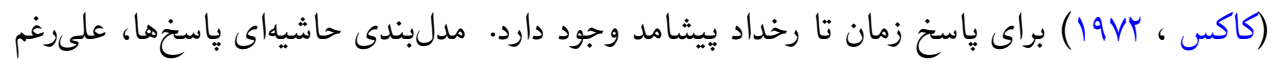

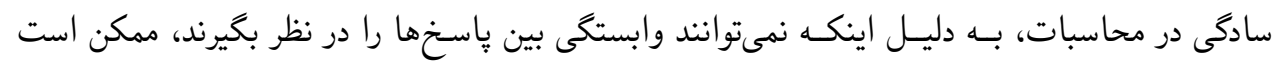

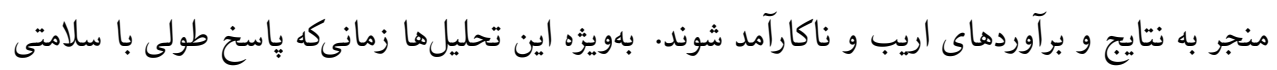

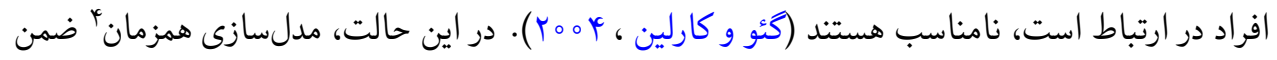

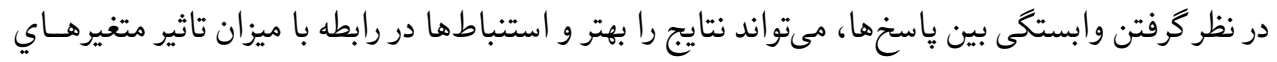

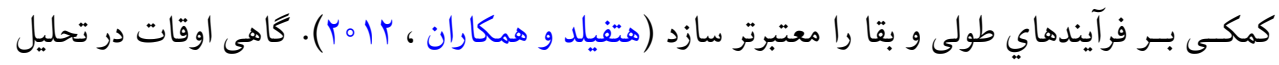

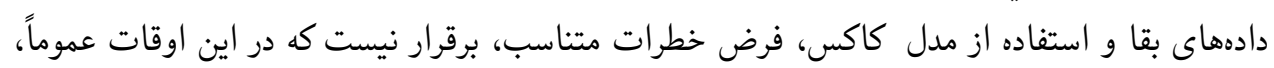

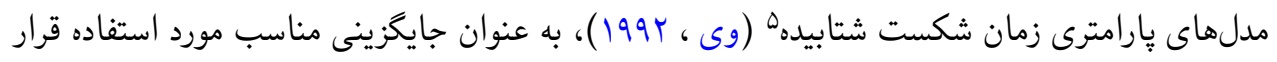

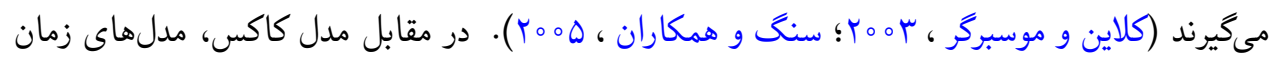

${ }^{1}$ Time to event

${ }^{2}$ Survival or Failure time

${ }^{3}$ Cox proportional hazards

${ }^{4}$ Simultaneous

${ }^{5}$ Accelerated Failure Time (AFT) 
شكست شتابيده مىتوانند بهترين مشخصسازى و تفسير عوامل ريسك (خطر) روى زمان شكست را

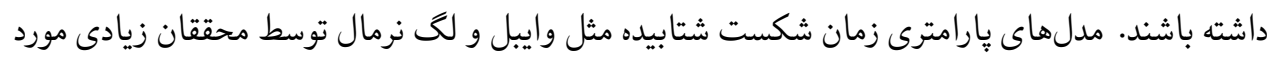

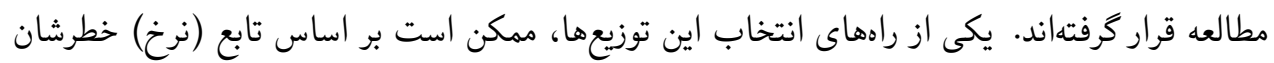

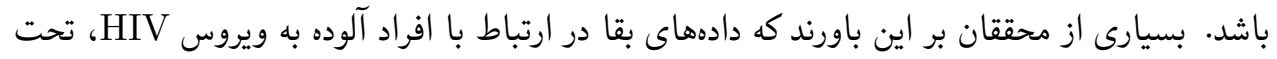

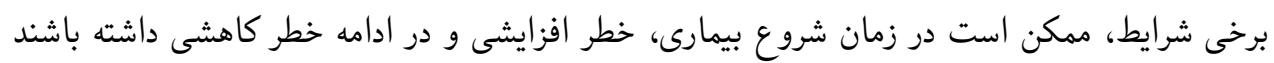

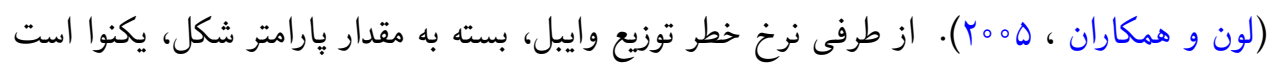

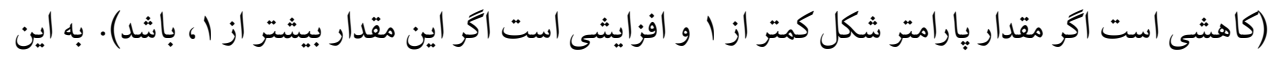

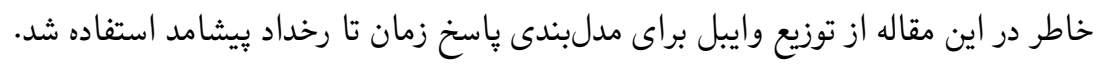
فرض كنيد $x_{i}=\left(1, x_{i 1}, \ldots, x_{i p}\right)^{\prime}{ }^{\prime}$ براى

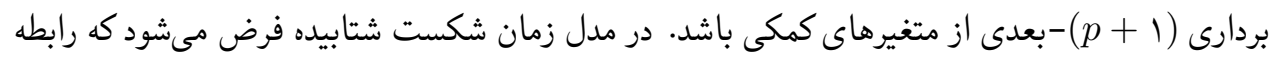
خطى بين لكاريتم

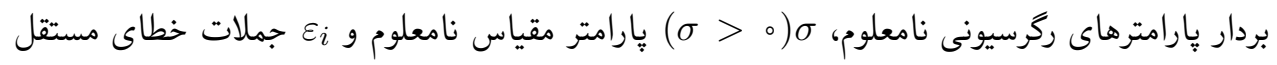

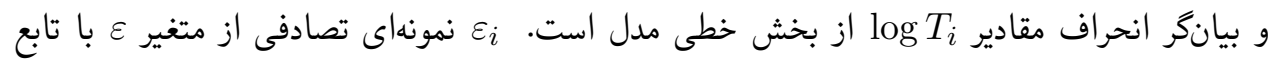
ج جالى معلوم (ك)

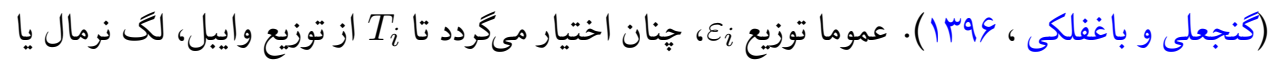

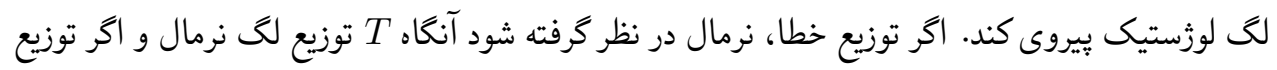

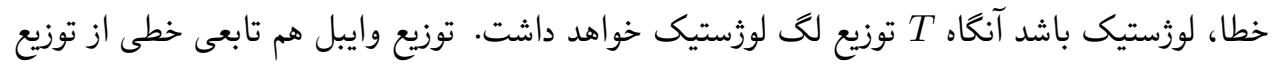

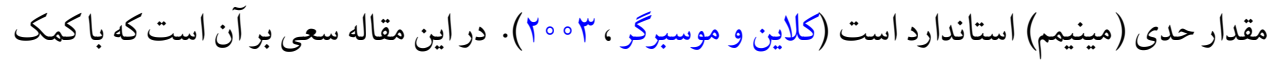

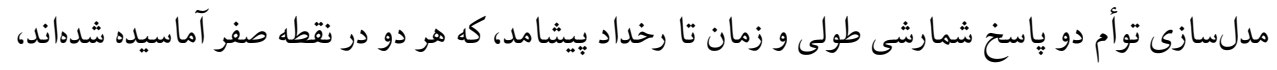

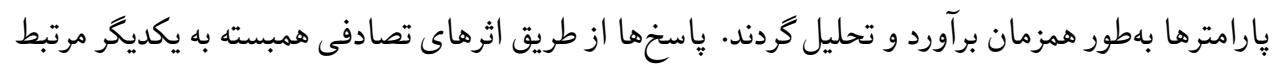

در بخش ץ جارجوب نظرى مدلبندى شامل مدل ركرسيون آميخته خطى تعميميافته براى ياسخ شمارشى

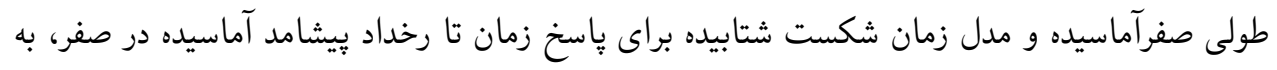

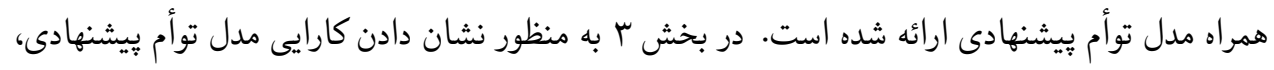

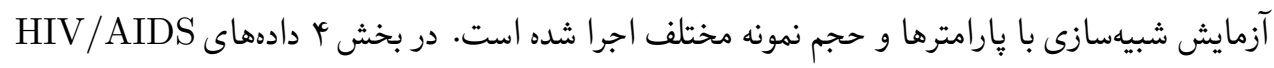

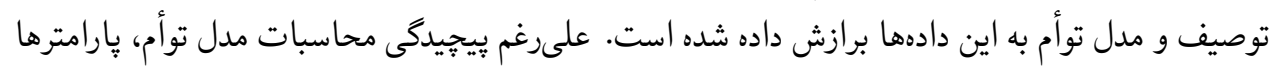
برآورد و خطاهاى استاندارد محاسبه شدهاند. در نهايت بحث و نتيجهيرى ارئ دائه شده است. 


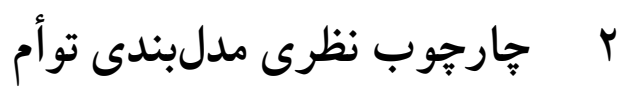

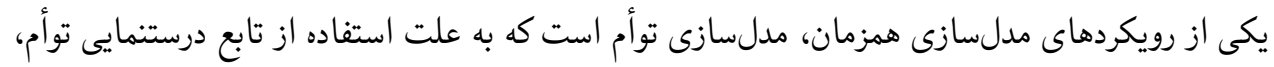

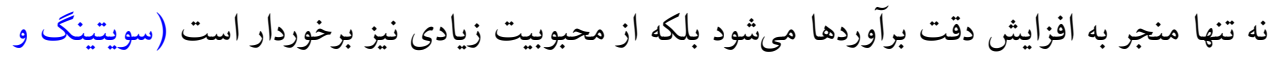

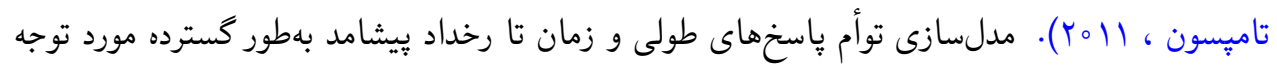

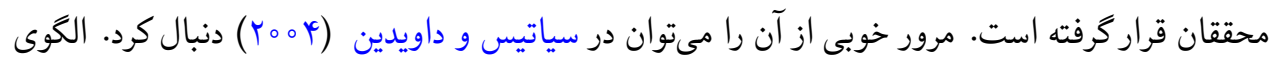

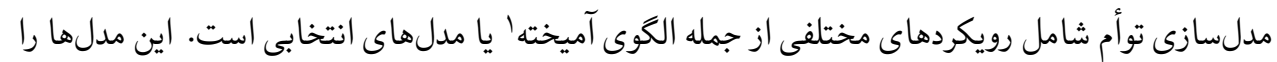

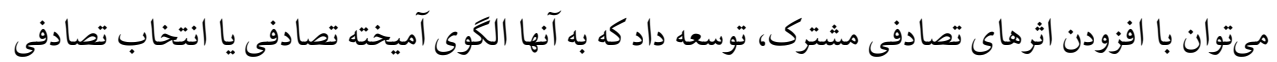

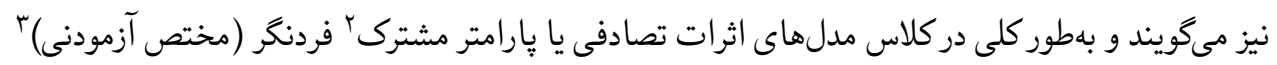

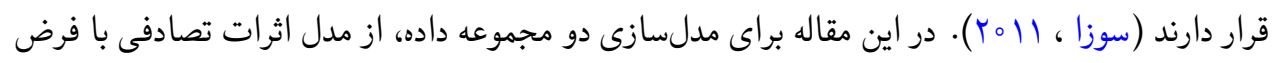

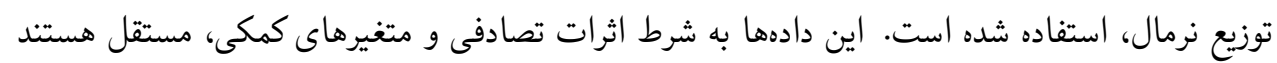

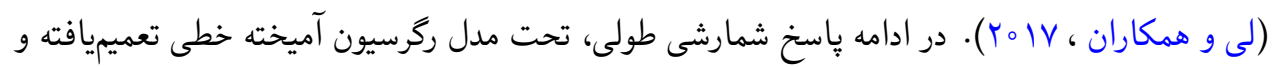

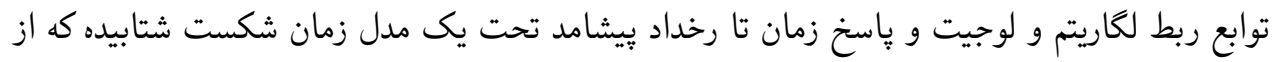

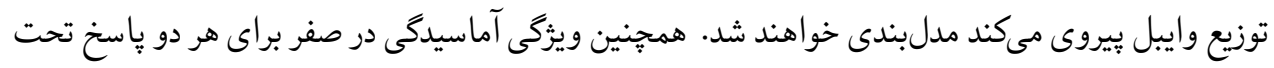

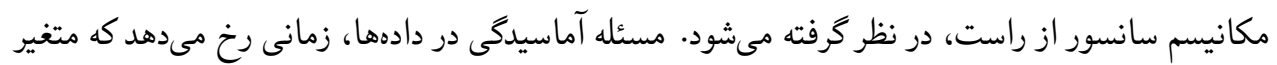

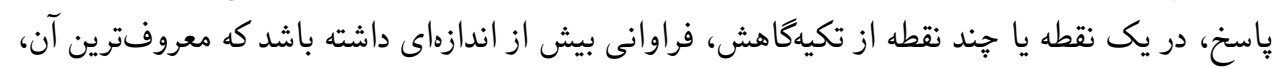

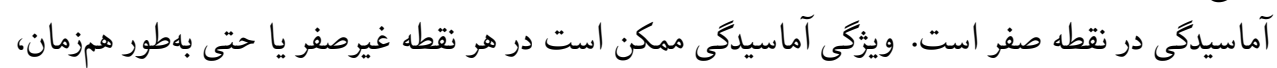

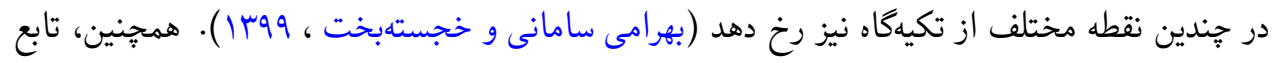

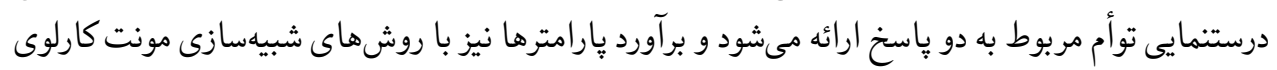

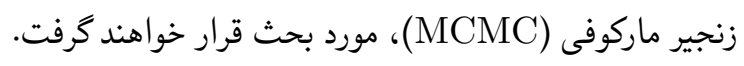

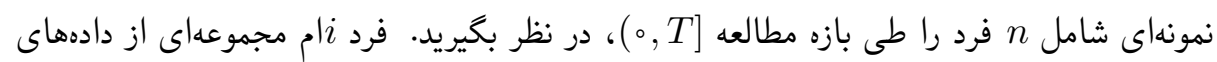
طولى (t)

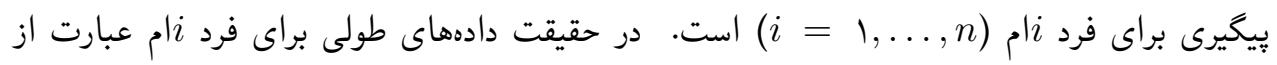
فض فرض كنيد

${ }^{1}$ Pattern-Mixture

${ }^{2}$ Shared Parameter

${ }^{3}$ Subject-Specific 


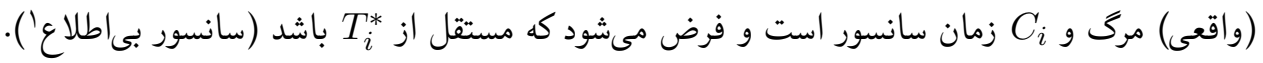

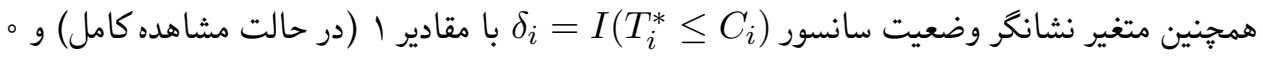

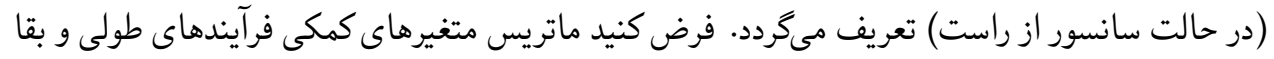

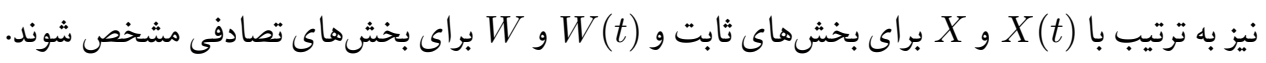

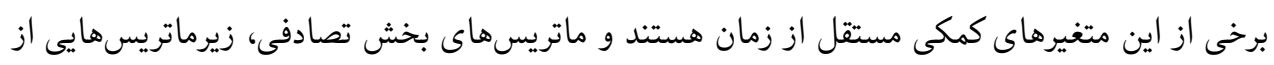
ماتريسهاى بخش ثابت در نظر كرفته مىشوند.

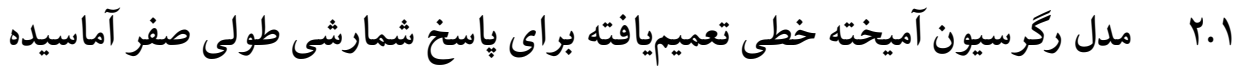

مدلهاى خطى تعميميافته در مواقعى كه ياسخ مورد نظر توزيع نرمال ندارد مورد استفاده قرار مىگيرند.

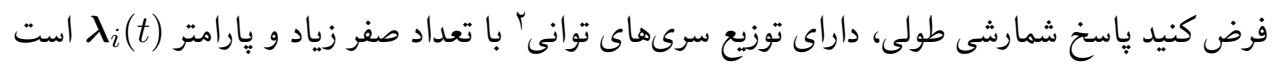

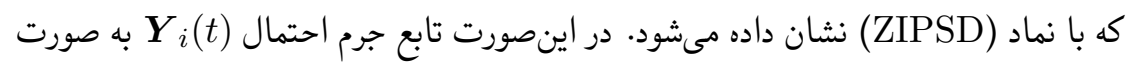

$$
P\left(\boldsymbol{Y}_{i}(t)=\boldsymbol{y}_{i}(t) ; \boldsymbol{\lambda}_{i}(t)\right)= \begin{cases}\boldsymbol{\pi}_{\backslash i}(t)+\left(1-\boldsymbol{\pi}_{\backslash i}(t)\right) \frac{a_{\circ}}{b\left(\lambda_{i}(t)\right)} & \boldsymbol{y}_{i}(t)=。 \\ \left(1-\boldsymbol{\pi}_{\backslash i}(t)\right) \frac{a_{y_{i}(t)}\left(\lambda_{i}(t)\right)^{y_{i}(t)}}{b\left(\lambda_{i}(t)\right)} & \boldsymbol{y}_{i}(t)>。\end{cases}
$$

است، كه در آن ه صفرآماسيده است كه احتمال آميختخى بين توزيع سرى توانى و و توزيع تباهيده در صفر را نشان مى اندهد

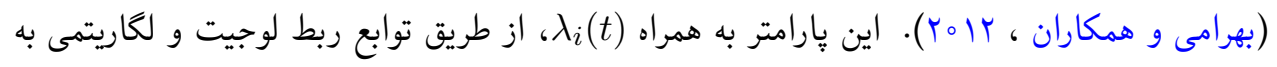

$$
\text { متغيرهاى كمكى مرتبط هستند. يعنى }
$$

$$
\begin{aligned}
\operatorname{logit}\left(\boldsymbol{\pi}_{\backslash i}(t)\right) & =\boldsymbol{X}_{\backslash i}^{\prime}(t) \boldsymbol{\beta}_{\backslash}+\boldsymbol{W}_{\backslash i}^{\prime}(t) \boldsymbol{\eta}_{\backslash i} \\
\log \left(\boldsymbol{\lambda}_{i}(t)\right) & =\boldsymbol{X}_{\curlyvee i}^{\prime}(t) \boldsymbol{\beta}_{\curlyvee}+\boldsymbol{W}_{\curlyvee i}^{\prime}(t) \boldsymbol{\eta}_{\curlyvee i}
\end{aligned}
$$

كه در آن متغيرهاى كمكى زمان-وابسته و و ضرائب ركرسيونى بخش ثابت مدل طولى هستند. بردارهاى (t)

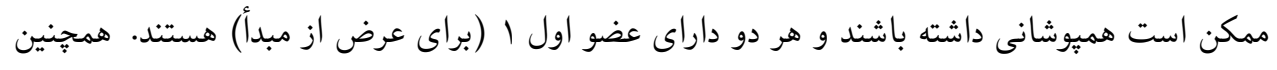

\footnotetext{
${ }^{1}$ Non-Informative

${ }^{2}$ Power Series Distribution (PSD)

${ }^{3}$ Time-Dependence (Time-Varying)
} 


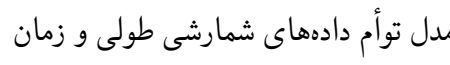

$\boldsymbol{W}_{\ell i}(t)$ (ب) هستند ( ) آن

$$
\mathbf{Y}_{i}(t) \mid \boldsymbol{\eta}_{i} ; \boldsymbol{\beta} \sim Z \operatorname{ZIPSD}\left(\boldsymbol{\lambda}_{i}(t), \boldsymbol{\pi}_{\backslash i}(t)\right) . \quad i=1, \ldots, n
$$

همجنين، تابع درستمايى شرطى دادهاى مشاهده شده، (t)

$$
L \boldsymbol{y}\left(\boldsymbol{y}_{i}(t) \mid \boldsymbol{\eta}_{i}\right)=P_{L C}\left(\boldsymbol{Y}_{i}(t)=\boldsymbol{y}_{i}(t) \mid \boldsymbol{\eta}_{i} ; \boldsymbol{\beta}\right)
$$

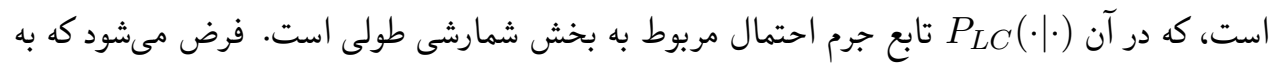

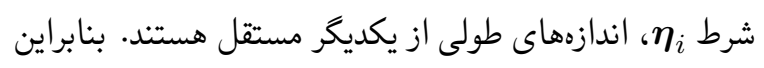

$$
P_{L C}\left(\boldsymbol{Y}_{i}(t)=\boldsymbol{y}_{i}(t) \mid \boldsymbol{\eta}_{i} ; \boldsymbol{\beta}\right)=\prod_{j} P\left(Y_{i}\left(t_{i j}\right)=y_{i}\left(t_{i j}\right) \mid \boldsymbol{\eta}_{i} ; \boldsymbol{\beta}\right)
$$

كه در آن ZIPS

$$
\begin{aligned}
& \left(P\left(Y_{i}\left(t_{i j}\right)=\circ \mid \boldsymbol{\eta}_{i} ; \boldsymbol{\beta}\right)\right)^{I\left(Y_{i}\left(t_{i j}\right)=\circ\right)}\left(P\left(Y_{i}\left(t_{i j}\right)=y_{i}\left(t_{i j}\right) \mid \boldsymbol{\eta}_{i} ; \boldsymbol{\beta}\right)\right)^{I\left(Y_{i}\left(t_{i j}\right)>\circ\right)} \\
= & \left\{\pi_{\backslash i}\left(t_{i j}\right)+\left(1-\pi_{\backslash i}\left(t_{i j}\right)\right) \frac{a_{\circ}}{b\left(\lambda_{i}\left(t_{i j}\right)\right)}\right\}^{I\left(Y_{i}\left(t_{i j}\right)=\circ\right)} \\
\times & \left\{\left(1-\pi_{\backslash i}\left(t_{i j}\right)\right) \frac{a_{y_{i}\left(t_{i j}\right)}\left(\lambda_{i}\left(t_{i j}\right)\right)^{y_{i}\left(t_{i j}\right)}}{b\left(\lambda_{i}\left(t_{i j}\right)\right)}\right\}^{I\left(Y_{i}\left(t_{i j}\right)>\circ\right)}
\end{aligned}
$$

r.r مدل زمان شكست شتابيده براى ياسخ زمان تا رخداد بيشامد صفر آماسيده براى فرآيند بقا يك مدل ركرسيون زمان شكست شتابيده با اثرات آميخته به صورت $\log \left(T_{i}^{*} \mid \boldsymbol{\vartheta}_{\uparrow i} ; \boldsymbol{\gamma}_{\uparrow}, \sigma\right)=\boldsymbol{X}_{r_{i}}^{\prime} \boldsymbol{\gamma}_{\uparrow}+\boldsymbol{W}_{r_{i}}^{\prime} \boldsymbol{\vartheta}_{\uparrow i}+\sigma \varepsilon_{i}$ 
را در نظر بخيريد، كه در آن

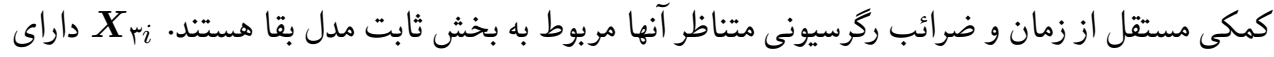

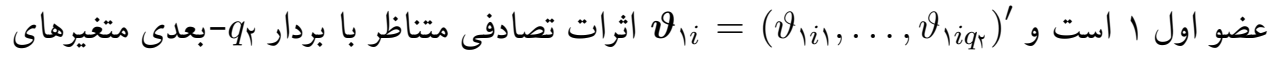
كمكى

$$
F\left(t_{i} \mid \boldsymbol{\vartheta}_{\backslash i}, \boldsymbol{\vartheta}_{\uparrow i} ; \boldsymbol{\gamma}_{1}, \boldsymbol{\gamma}_{\uparrow}, \alpha\right)=\pi_{\uparrow i}+\left(1-\pi_{\uparrow i}\right) F_{T^{*}>\circ}\left(t_{i} \mid \boldsymbol{\vartheta}_{\backslash i} ; \gamma_{1}, \alpha\right)
$$

است، كه در آن

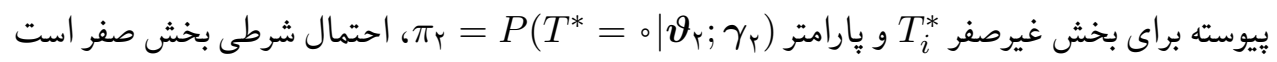

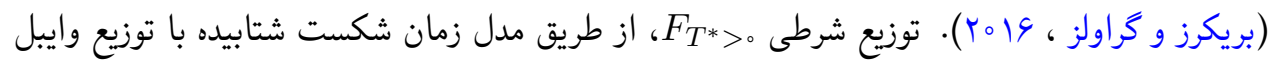

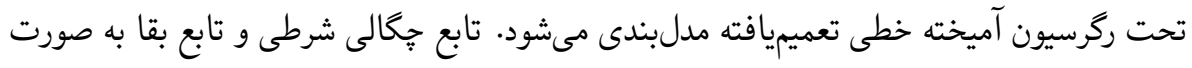

$$
\begin{aligned}
& f_{T^{*}>\circ}\left(t \mid \boldsymbol{\vartheta}_{1} ; \gamma_{1}, \alpha\right)=\lambda^{*} \alpha t^{\alpha-1} \exp \left(-\lambda^{*} t^{\alpha}\right) \\
& S_{T^{*}>\circ}\left(t \mid \boldsymbol{\vartheta}_{1} ; \gamma_{1}, \alpha\right)=e^{-\lambda^{*} t^{\alpha}}
\end{aligned}
$$

خواهند بود، كه در آن ركرسيونى لوزستيك و و و $\boldsymbol{\vartheta}_{\text {I }}$

$$
\text { داده شود }
$$

$$
T_{i} \mid \boldsymbol{\vartheta}_{i} ; \gamma, \alpha \sim Z I W \operatorname{eiAFT}\left(\alpha, \lambda_{i}^{*}, \pi_{\uparrow i}\right)
$$

همجنين، تابع درستنمايى شرطى $t_{\text {و }}$ ؛ مربوط به iامين فرد، s s ، در حالت سانسور از راست به صورت

$$
\begin{aligned}
L_{s}\left(t_{i}, \delta_{i} \mid \boldsymbol{\vartheta}_{i}\right) & =\left[f\left(t_{i} \mid \boldsymbol{\vartheta}_{i} ; \boldsymbol{\gamma}, \alpha\right)\right]^{\delta_{i}}\left[S\left(t_{i} \mid \boldsymbol{\vartheta}_{i} ; \boldsymbol{\gamma}, \alpha\right)\right]^{1-\delta_{i}} \\
& =\left[\left(1-\pi_{\curlyvee i}\right) f_{T^{*}>\circ}\left(t_{i} \mid \boldsymbol{\vartheta}_{i} ; \boldsymbol{\gamma}, \alpha\right)\right]^{\delta_{i}}\left[\left(1-\pi_{\curlyvee i}\right)\left(1-F_{T^{*}>\circ}\left(t_{i} \mid \boldsymbol{\vartheta}_{i} ; \boldsymbol{\gamma}, \alpha\right)\right)\right]^{1-\delta_{i}} \\
& =\left(1-\pi_{\curlyvee i}\right)\left[f_{T^{*}>\circ}\left(t_{i} \mid \boldsymbol{\vartheta}_{i} ; \boldsymbol{\gamma}, \alpha\right)\right]^{\delta_{i}}\left[S\left(t_{i} \mid \boldsymbol{\vartheta}_{i} ; \boldsymbol{\gamma}, \alpha\right)\right]^{1-\delta_{i}}
\end{aligned}
$$




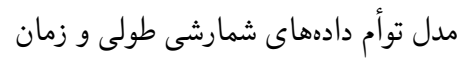

$$
\begin{aligned}
& \frac{d F\left(t_{i} \mid \boldsymbol{\vartheta}_{i} ; \gamma, \alpha\right)}{d t_{i}}=\left(1-\pi_{\ulcorner i}\right) \frac{d F_{T^{*}>\circ}\left(t_{i} \mid \boldsymbol{\vartheta}_{\backslash i} ; \boldsymbol{\gamma}_{\curlywedge}, \alpha\right)}{d t_{i}} \\
& \text { و همجنين (·) } f_{T^{*}>\circ}(t \mid \cdot)=d / d t F_{T^{*}>0}(t) \text { (كلاين و موسبركر ، roor) }
\end{aligned}
$$

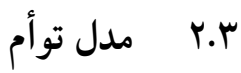

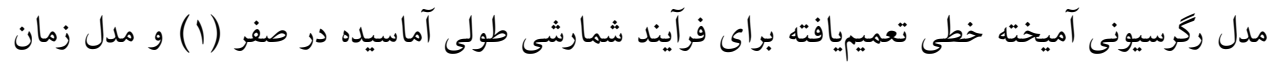

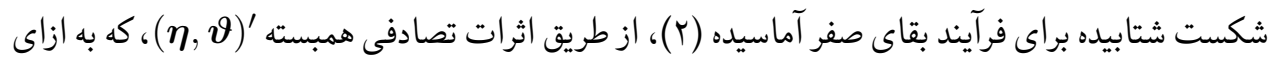

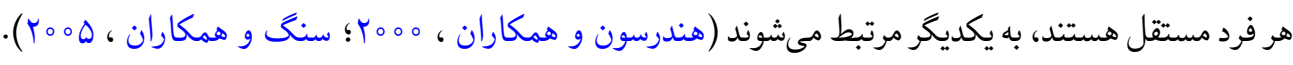
در اينجا، فرض مىشود (هر تصادفى دو مولفه طولى و بقا است و به صورت

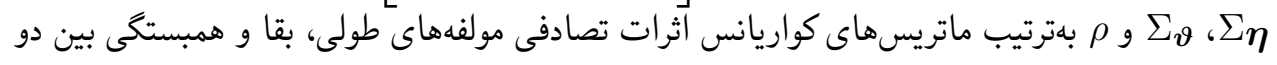

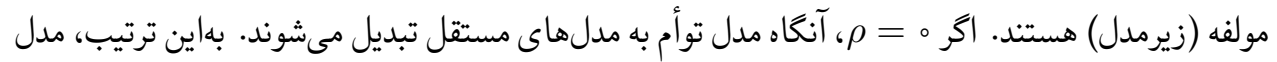

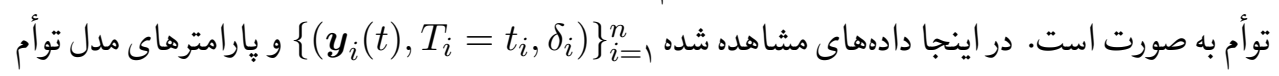

$$
\begin{aligned}
& \mathbf{Y}_{i}(t)\left|\boldsymbol{\eta}_{i} ; \boldsymbol{\beta} \sim \operatorname{ZIPSD}\left(\lambda_{i}(t), \pi_{\|_{i}}(t)\right) \quad T_{i}\right| \boldsymbol{\vartheta}_{i} ; \boldsymbol{\gamma}, \alpha \sim Z \operatorname{IWeiAFT}\left(\alpha, \lambda_{i}^{*}, \pi_{r i}\right) \\
& \log \left(\lambda_{i}(t)\right)=\boldsymbol{X}_{r_{i}}^{\prime}(t) \boldsymbol{\beta}_{\curlyvee}+\boldsymbol{W}_{r_{i}}^{\prime}(t) \boldsymbol{\eta}_{\curlyvee i} \quad \log \left(\lambda_{i}^{*}\right)=-\alpha\left\{\boldsymbol{X}_{r_{i}}^{\prime} \boldsymbol{\gamma}_{\uparrow}+\boldsymbol{W}_{r_{i}}^{\prime} \boldsymbol{\vartheta}_{\uparrow i}\right\} \\
& \operatorname{logit}\left(\pi_{\backslash i}(t)\right)=\boldsymbol{X}_{\backslash i}^{\prime}(t) \boldsymbol{\beta}_{\uparrow}+\boldsymbol{W}_{\backslash i}(t)^{\prime} \boldsymbol{\eta}_{\backslash i} \quad \operatorname{logit}\left(\pi_{\curlyvee i}\right)=\boldsymbol{X}_{\nvdash_{i}}^{\prime} \boldsymbol{\gamma}_{\uparrow}+\boldsymbol{W}_{\nvdash_{i}}^{\prime} \boldsymbol{\vartheta}_{\curlyvee i} \\
& \text { هستند. در اينصورت، تابع درستنمايى مدل توأم به صورت } \Psi=\left(\boldsymbol{\beta}, \boldsymbol{\gamma}, D_{\boldsymbol{\eta}, \boldsymbol{\vartheta}}, \sigma\right) \\
& L(\boldsymbol{\Psi} ; \boldsymbol{y}(t), t, \delta)=\prod_{i=1}^{n} \int L_{y}\left(\boldsymbol{y}_{i}(t) \mid \boldsymbol{\eta}_{i}\right) L_{s}\left(t_{i}, \delta_{i} \mid \boldsymbol{\vartheta}_{i}\right) f\left(\boldsymbol{\eta}_{i}, \boldsymbol{\vartheta}_{i}\right) d \boldsymbol{\eta}_{i} d \boldsymbol{\vartheta}_{i}
\end{aligned}
$$

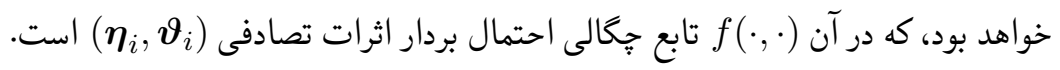




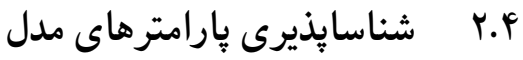

شناسايذيرى مدلهاى آمارى، يكى از مهمترين ويزگىهاى پارامترهاى يك مدل آمارى مناسب است. اكر

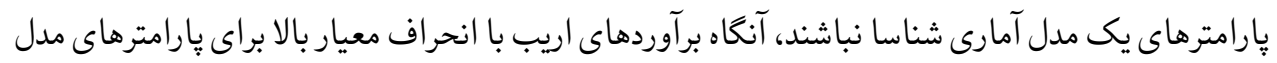

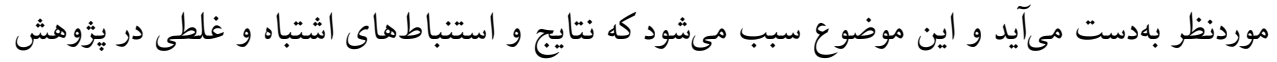

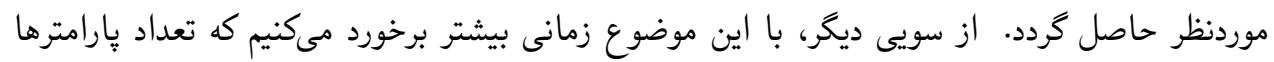

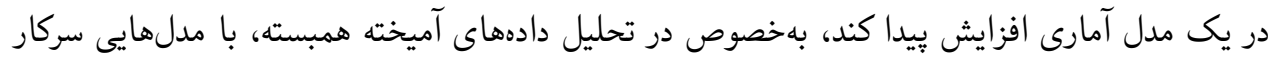

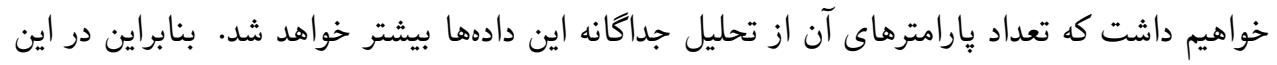

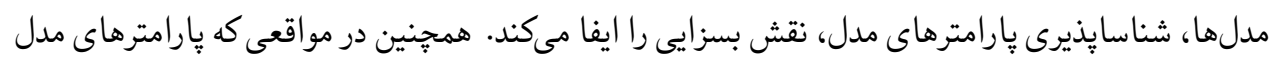

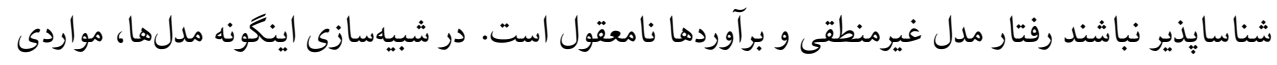

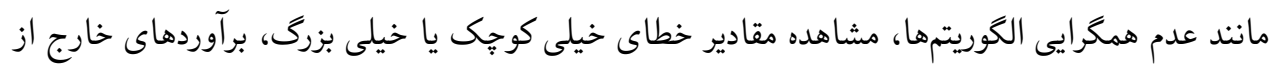
دامنه فضاى بارامتر، فواصل اطمينان با طول زياد مشاهده مىشود و و بعضا با تغيير نقطه اوليه، برآوردها

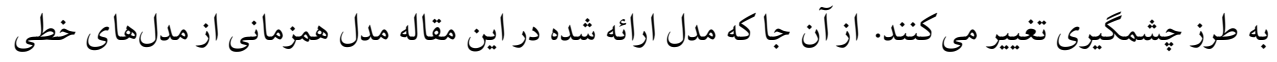

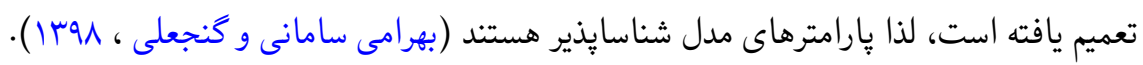

\section{r ب م مالعه شبيهسازى}

در اين بخش براى نشان دادن عملكرد و كارايى مدل توأم ييشنهادى كه در بخش قبل ارائه شده است،

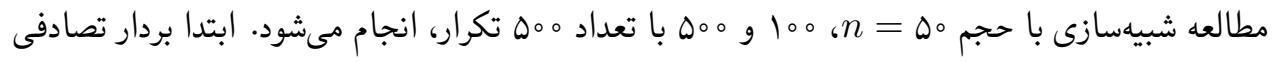

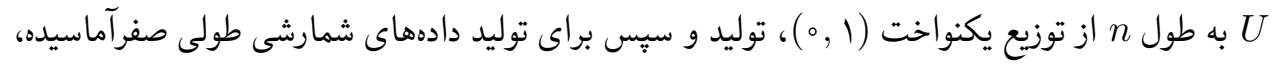

$$
\text { تبه }
$$

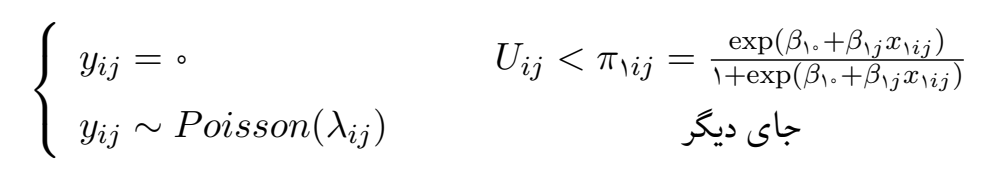

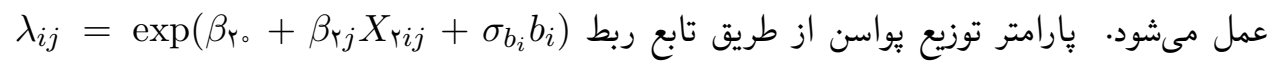

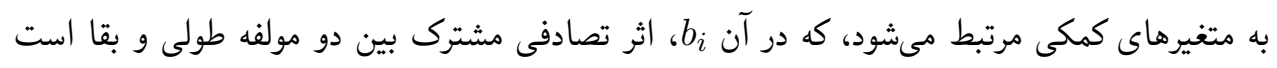
بلهورىكه 
مدل توأم دادهاى شمارشى طولى و زمان

شكست شتابيده،

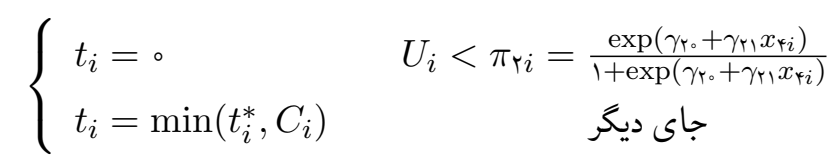

عمل مىشود، كه در آن i از ازتوزيع مقدار حدى شبيهسازى مىشود. در اين فرآيند، مقادير اوليه بارامترها عبارتاند از: ز از

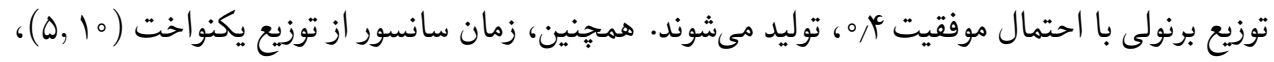

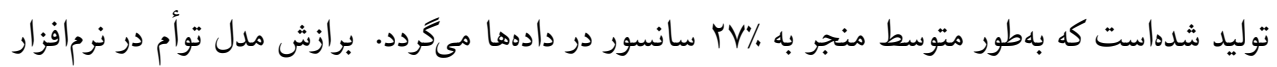

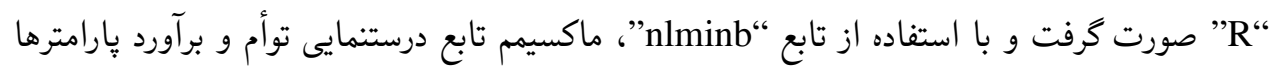

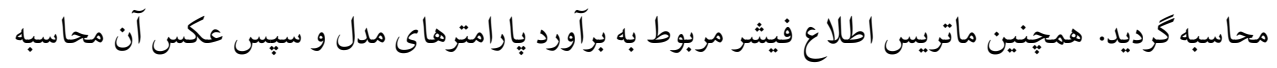

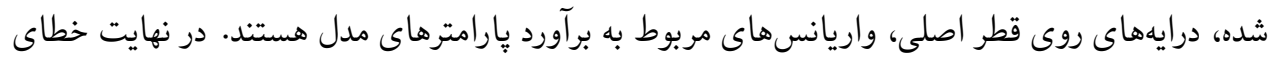

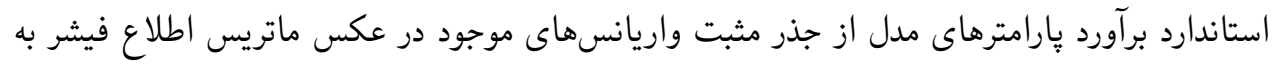

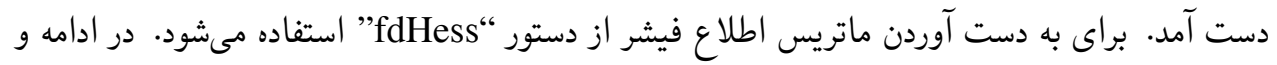

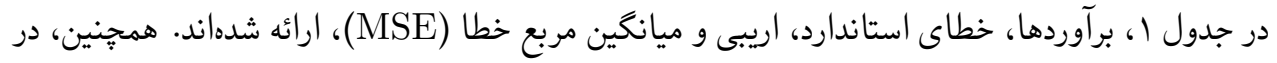

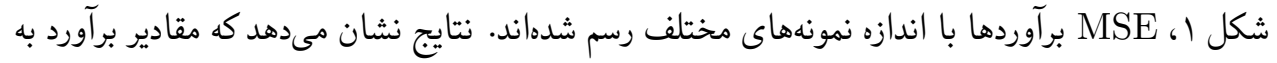

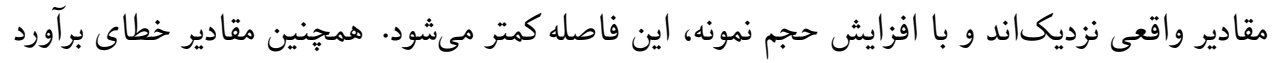

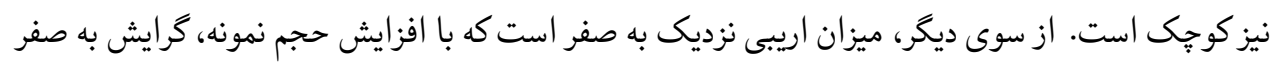

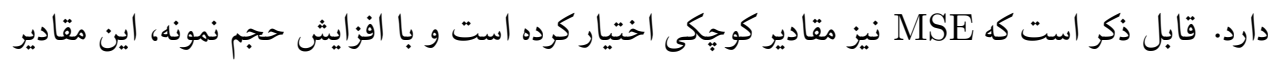

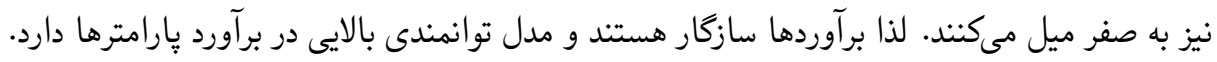

\section{HIV / AIDS تحليل دادههاى}

در اين بخش مدل توأم ارائه شده را روى مجموعه دادههايى كه توسط كلدمن و همكاران (1999) جمع آورى

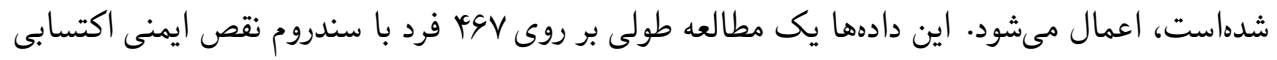

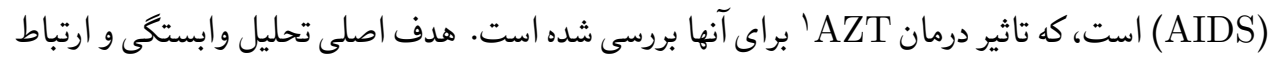




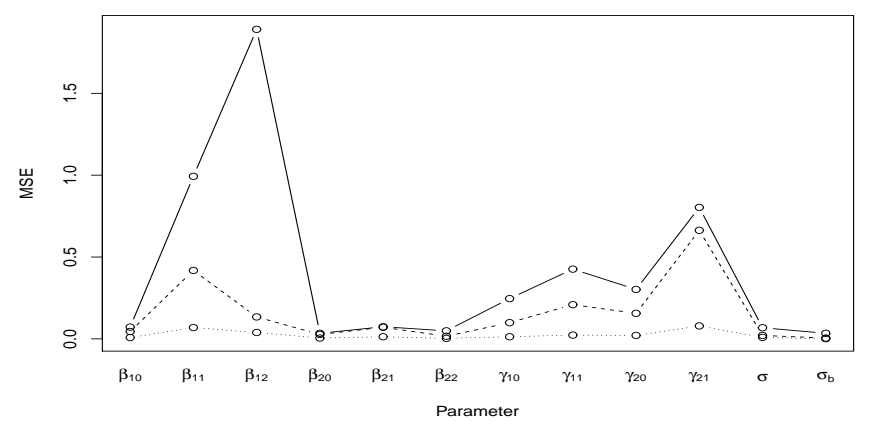

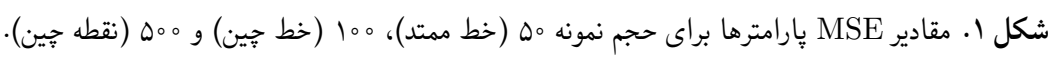

بين تعداد سلولهاى CD4 و زمان مرى افراد و مقايسه اثربخشى و اطمينان به دو داروى ضدويروسى

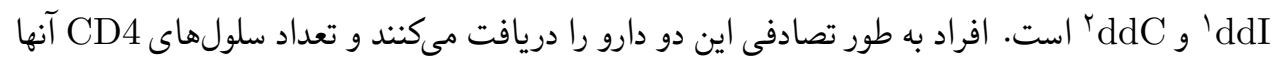

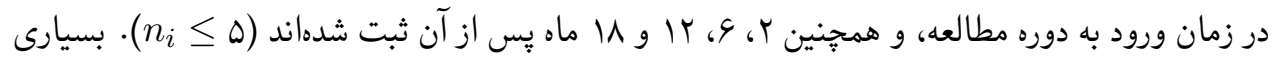

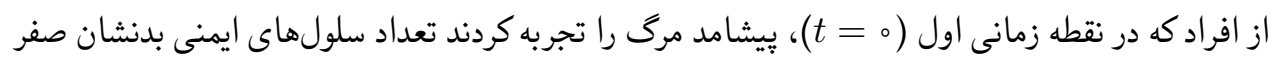

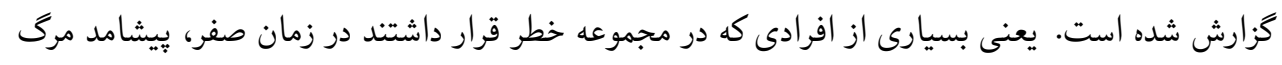

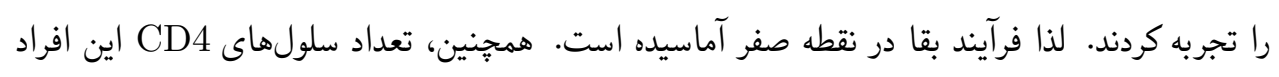

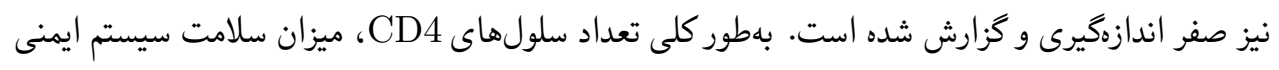

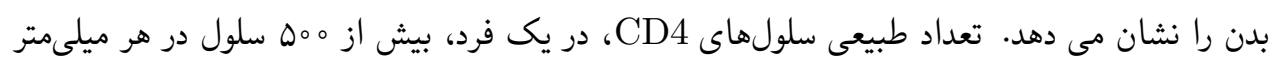

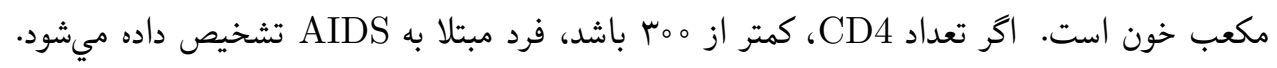

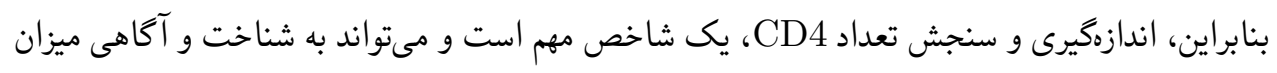

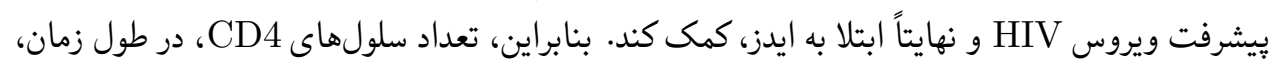

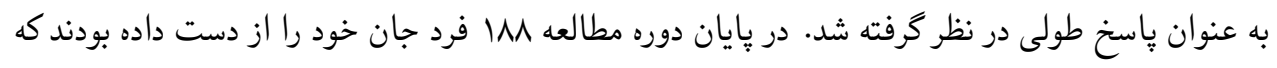

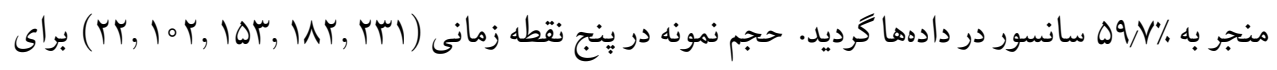

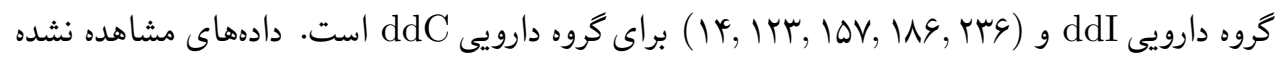
با كذشت زمان به علت مرگ و مير، انصراف و ترك مطالعه و عدم مراجعه به درمانكاه، به شدت درو درو دال دال

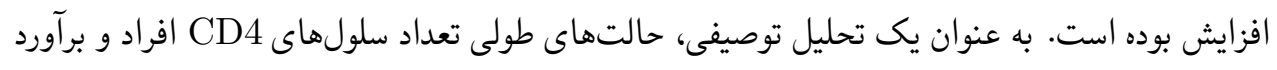

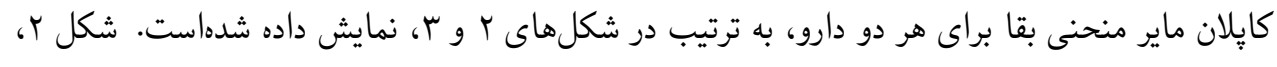


مجتبى زينلى و احسان بهرامى .

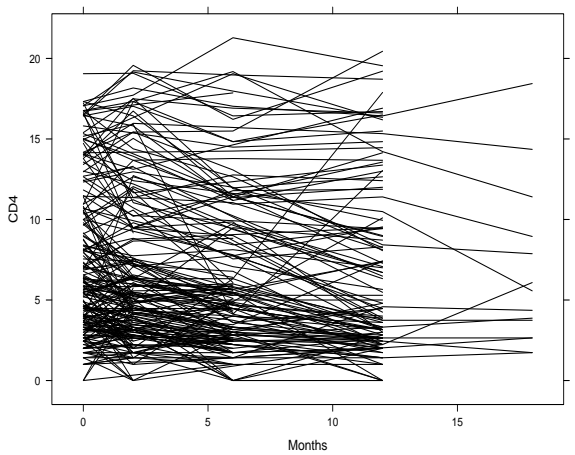

$(ب)$

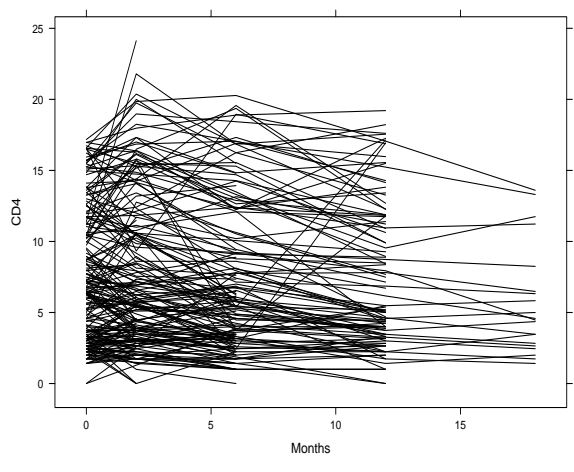

(الف) شكل r. تغييرات تعداد سلولهاى CD4 افراد، براى دو داروى الف: ddI و ب: ddC.

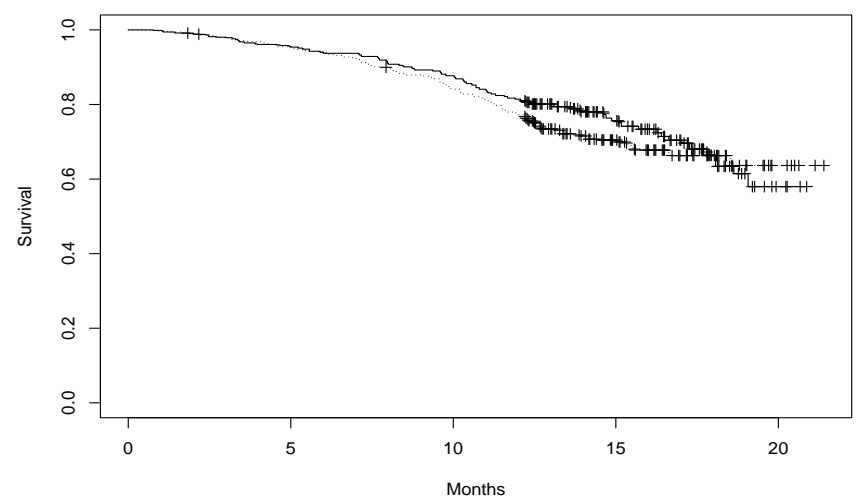

شكل r. برآوردهاى كايلان ماير احتمال بقا براى ddI (نقطهجين) و ddC (خط ممتد).

نرخ بالايى از گمشدكى را در طول زمان به تفكيك دو داروى ضد ويروسى نشان مىدهد. همدينين باتوجه

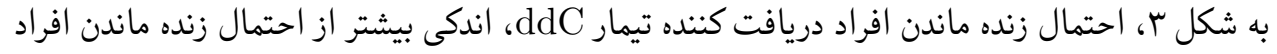

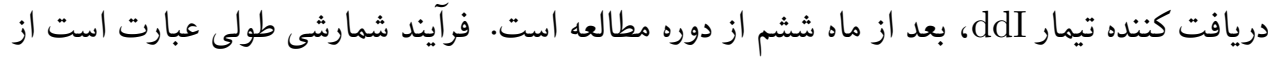

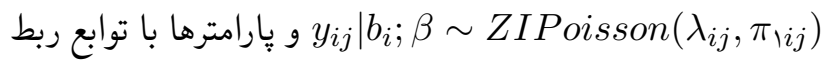

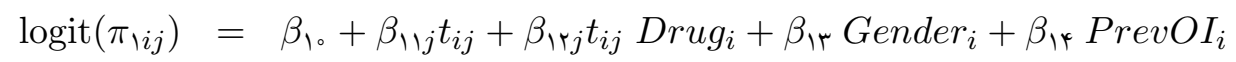


مدل توأم دادهاى شمارشى طولى و زمان

$$
\begin{aligned}
& +\beta_{\iota_{\Delta}} A Z T_{i} \\
\log \left(\lambda_{i j}\right) & =\beta_{\Upsilon^{\circ}}+\beta_{\Upsilon \backslash j} t_{i j}+\beta_{\Upsilon \Upsilon j} t_{i j} \text { Drug }_{i}+\beta_{\Upsilon \Upsilon} \text { Gender }_{i}+\beta_{\Upsilon \uparrow} \text { PrevOI }_{i} \\
& +\beta_{\Upsilon \triangleright} A Z T_{i}+\sigma_{b_{i}} b_{i}
\end{aligned}
$$

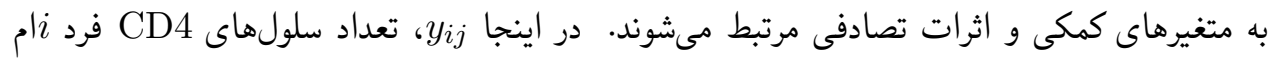

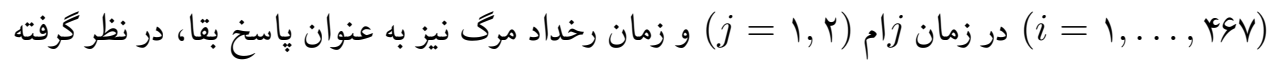

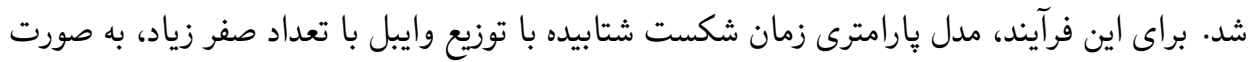

$$
\begin{aligned}
& T_{i} \mid b_{i} ; \gamma, \alpha \sim Z I W \operatorname{eiAFT}\left(\alpha, \lambda_{i}^{*}, \pi_{\uparrow i}\right)
\end{aligned}
$$

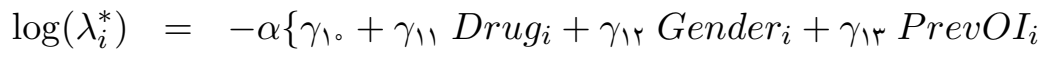

$$
\begin{aligned}
& \left.+\gamma_{1 \uparrow} A Z T_{i}+\sigma_{b_{i}} b_{i}\right\}
\end{aligned}
$$

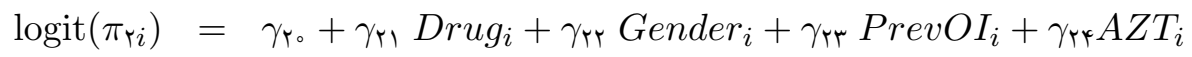

است. در مجموعه دادهها Gender

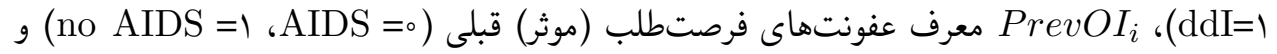

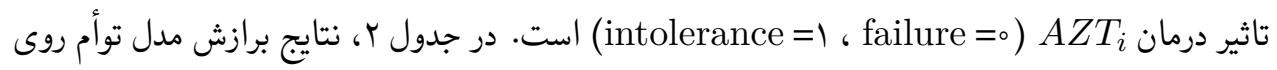

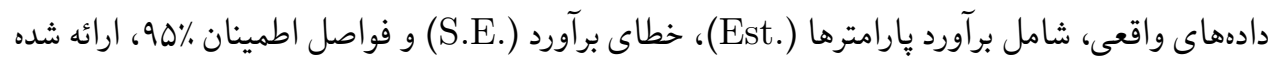

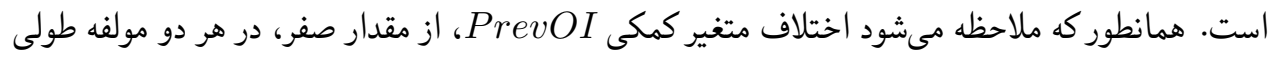

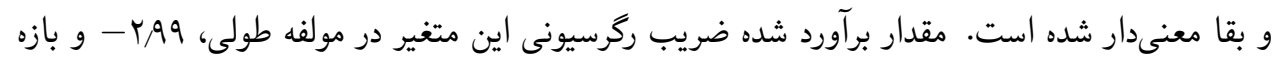

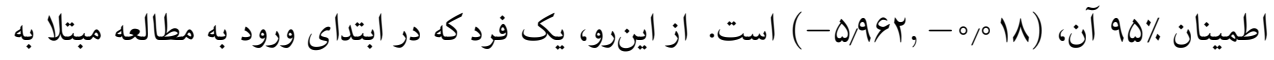

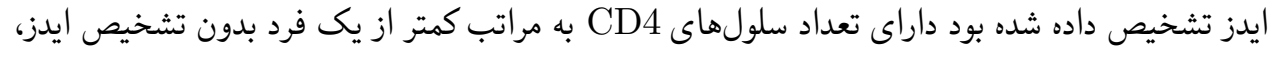
بوده است. همجنين، اختلاف معنىدارى بين دو نوع دارو (ddC و ddI) وجود ندود ندارد.

\section{بحث و نتيجهگيرى}

براى تحليل دادههاى حاصل از دو ياسخ همبسته طولى و زمان تا رخداد بيشامد، كه هر دو در نقطه صفر

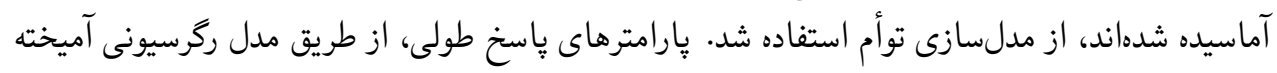


خطى تعميميافته، به متغيرهاى كمكى مرتبط شدند. جون اكثر مطالعات بقا، بيانكر آن است كه برآورد

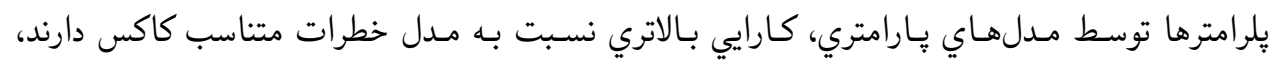

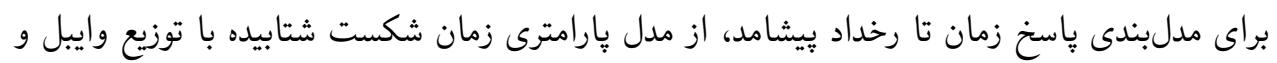

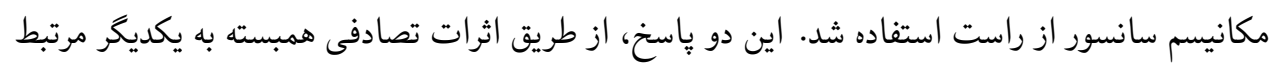

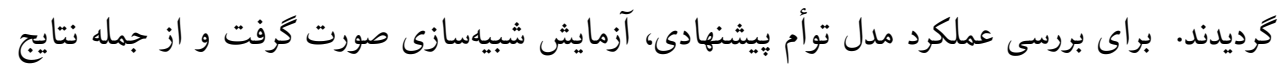

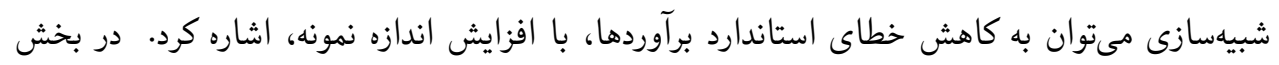

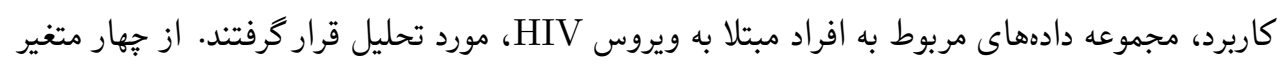

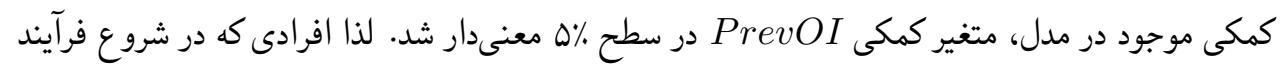

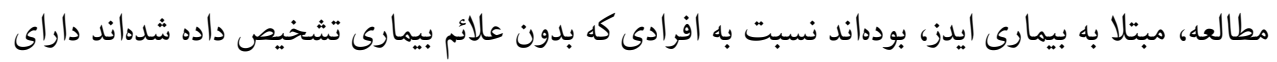

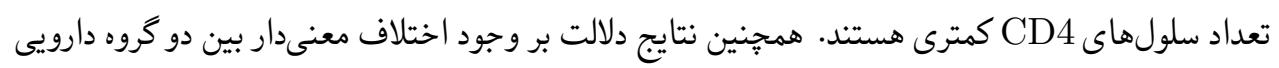

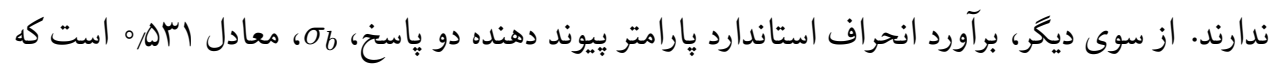

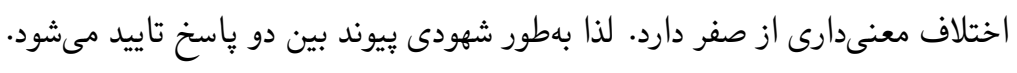

\section{تقدير و تشكر}

از داوران و ويراستار محترم مجله كه با توصيههاى ارزشمند خود سبب ارتقاى اين مقاله و ارائه بهتر آن شدهاند كمال تشكر را داريم.

\section{مراجع}

بهرامى سامانى، ا. و خجستهبخت، ن. (1\%99)، تحليل نيميارامترى مدلهاى ركرسيونى براى پاسخهاى

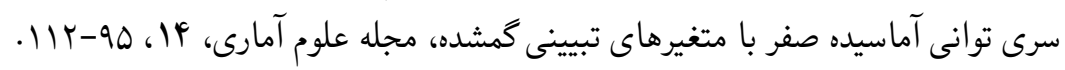

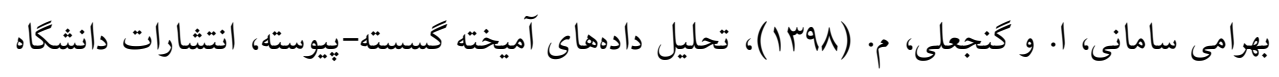
شهيد بهشتى تهران، تهران.

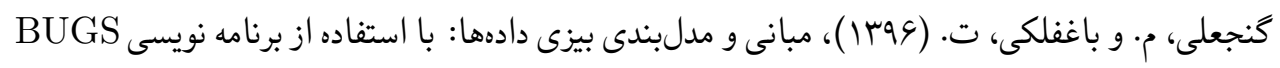
و نرمفزار R، انتشارات دانشعاه شهيد بهشتى، تهران.

Bahrami, S. E., Ganjali, M. and Amirian, Y. (2012), Likelihood Estimation 
for Longitudinal Zero-Inflated Power Series Regression Models, Journal of Applied Statistic, 39, 1965-1974.

Braekers, R. and Grouwels, Y., (2016), A Semi-Parametric Cox's Regression Model for Zero Inflated Left-Censored Time To Event Data, Communications in Statistics - Theory and Methods, 45, 1969-1988.

Cox, D. R. (1972), Regression Models and Life-Tables, Journal of the Royal Statistical Society, Series B, 34: 187-220.

Goldman, A. I., Carlin, B. P., Crane, L. R., Launer, C., Korvick, J. A., Deyton, L. and Abrams, D. I. (1996), Response of CD4+ and Clinical Consequences to Treatment Using ddI or ddC in Patients with Advanced HIV Infection, Journal of Acquired Immune Deficiency Syndromes and Human Retrovirology, 11: 161-169.

Guo, X. and Carlin, B. P. (2004), Separate and Joint Modeling of Longitudinal and Event Time Data Using Standard Computer Packages, Journal American Statistical Association, 58: 16-24.

Hatfield, A., Hodges, S., and Carlin, P. (2012), Combining Longitudinal and Survival Information in Bayesian Joint Models: When Are Treatment Estimates Improved? Biostatistics, 0: 1-31.

Henderson, R., Diggle, P. and Dobson, A. (2000), Joint Modeling of Longitudinal Measurementsand Event Time Data, Biostatistics, 4:465-480.

Klein, J. P. and Moeschberger, M. L. (2003), SURVIVAL ANALYSIS- Techniques for Censored and Truncated Data, Second Edition, Statistics for Biology and Health, Springer LLC. 
Lawn, S. D., Myer, L., Orrell, C., Bekker, L. G. and Wood, R. (2005), Early Mortality Among Adult Saccessing a Community-Based Antiretroviral Service in South Africa: Implications for Programme Design, AIDS, 19, 2141-2148.

Li, S., Zheng, M. and Gao, S. (2017), Joint Modeling of Longitudinal Cholesterol Measurements and Time To Onset of Dementia in An Elderly African American Cohort, Biostatistics 85 Epidemiology, 1: 148-160.

Sousa, I. (2011), A Reviw on Joint Modelling of Longitudinal Measurements And Time-To-Event, REVSTAT Statistical Journal, 9: 57-81.

Sweeting, M. J. and Thompson, S. G. (2011), Joint Modelling of Longitudinal And Time-To-Event Data with Application to Predicting Abdominal Aortic Aneurysm Growth and Rupture, Biometrical Journal, 53: 750-763.

Tseng, Y., Hsieh, F. and Wang, J. L. (2005), Joint Modelling of Accelerated Failure Time and Longitudinal Data, Biometrika, 92: 587-603.

Tsiatis, A. A. and Davidian, M. (2004), Joint Modeling of Longitudinal and Time-To-Event Data: An Overview, Statistica Sinica, 14: 809-834.

Wei, L. J. (1992), The Accelerated Failure Time Model: A Useful Alternative to the Cox Regression Model in Survival Analysis, Stat Med, 11: 1871-9.

WHO, (2018), Global Health Observatory Data; Available at: www . who . int/ gho/hiv/epidemic_status/cases_all/en.

Wu, L., Liu, W., Grace, Y. and Hung, Y. (2012), Analysis of Longitudinal and Survival Data: Joint Modeling, Inference Methods, and Issues, Journal of Probability and Statistics. 1: 1-17. 
Journal of Statistical Sciences, Spring and Summer, 2021

Vol. 15, No. 1, pp 147-164

DOI: $10.29252 /$ jss.15.1.23

\title{
Joint Model of Longitudinal Count and Time to Event Data with Excess Zeros Using the AFT Model: A Case Study of the HIV / AIDS Dataset
}

Zeinali Najafabadi, M. and Bahrami Samani, E.

Department of Statistics, Shahid Beheshti University, Tehran, Iran.

\begin{abstract}
This article aims to joint modeling of longitudinal CDr cells count and time to death in HIV patients based on the AFT model. The modeling of the longitudinal count response, a GLME model under the family of PSD, was used. In contrast, for the TTE data, the parametric AFT model under the Weibull distribution was investigated. These two responses are linked through random effects correlated with the normal distribution. The longitudinal and survival data are then assumed independent, given the latent linking process and any available covariates. Considering excess zeros for two responses and right censoring, presented a joint model that has not yet been investigated by other researchers. The parameters were also estimated using MCMC methods.
\end{abstract}

Keywords: Accelerated failure time model, Time to event, Right censoring, Family of power series distribution, Generalized linear mixed effect (GLME) model.

Mathematics Subject Classification (2010): 62J05, 62J12. 